\title{
Großbölting, Thomas/Lenhard-Schramm, Niklas (Hrsg.): Contergan. Hintergründe und Folgen eines Arzneimittel-Skandals, 221 S., Vandenhoeck \& Ruprecht, Göttingen 2017.
}

\section{Livia Prüll}

Online publiziert: 27. November 2020

(C) Der/die Autor(en) 2020

Der „Fall Contergan“ bezeichnet einen der wohl bekanntesten Arzneimittelskandale in der Geschichte des 20. Jahrhunderts. Schon allein deshalb ist der Fall nicht abgeschlossen, und erst recht dann nicht, wenn man die Hintergründe und Auswirkungen mit in Betracht zieht. 1957 hatte die Aachener Pharmafirma Grünenthal mit der Substanz Thalidomid (Vertriebsname: „Contergan“) ein Arzneimittel auf den Markt gebracht, das als ,ungiftiges“ Schlafmittel letztlich dazu führte, dass mindestens 5000 Kinder mit zum Teil schwersten Extremitäten- und Organschädigungen zur Welt kamen. 1961 wurde das Mittel wieder vom Markt genommen.

Der vorliegende von Thomas Großbölting und Niklas Lenhard-Schramm herausgegebene Sammelband vereinigt acht Beiträge, die aus einer Tagung im September 2015 an der Westfälischen Wilhelms-Universität Münster hervorgegangen sind. In einer 20-seitigen Einleitung umreißen die Herausgeber zunächst das Thema. Neben einer kurzen Fallbeschreibung sprechen sie hier die weiteren Dimensionen des Vorfalls an, welche die Politik-, Sozial-, Mentalitäts- und Medizingeschichte der Bundesrepublik berühren. Die Zielsetzung des Bandes wird in zwei Punkten gebündelt: Erstens geht es um eine Behandlung der ,,sozialen, politischen und kulturellen Bedingungen“ (S. 14), die den Skandal ermöglichten; zweitens um die Frage, ob es sich bei dem Fall „Contergan“ um einen „Sonderfall“ handelte (S. 15). Die folgenden Aufsätze beziehen sich dann auch auf verschiedenste Aspekte des Themas. Behandelt werden im Einzelnen die medizin- und pharmaziehistorischen Hintergründe (Christoph Friedrich), der Umgang mit Gefahrenstoffen in den 1950er Jahren (Heiko Stoff), die Konsumgesellschaft der 1950er und beginnenden 1960er Jahre (Nils Kessel), die zeitgenössischen Diskussionen um „Arzneimittelnebenwirkungen“ (Anne H. Crumbach), die Haltung der Politik (Sabine Mecking), das Arzneimittelrecht (Niklas Lenhard-Schramm), eine Vergleichsstudie zum Thalidomid-haltigen Kombinationspräparat „Grippex“ (Ludger Wimmelbücker) und schließlich ein allgemeiner Beitrag zum gesellschaftlichen Umgang mit Arzneimittelrisiken (Hans-Jochen Luhmann).

\footnotetext{
L. Prüll $(\bowtie)$

Universitätsmedizin der Johannes Gutenberg-Universität Mainz, Mainz, Deutschland

E-Mail: pruell@uni-mainz.de
} 
Allen Beiträgen ist gemeinsam, dass sie das Thema auf die (frühe) Bundesrepublik fokussieren und aus dem Blickwinkel der Wissenschaftsgeschichte und Wissenschaftstheorie betrachten. Dabei werden zahlreiche Einzelerkenntnisse eingebracht, die im Rahmen der neueren historischen Arzneimittelforschung generiert wurden. Es gelingt so, ganz wesentliche Facetten des Skandals gut herauszuarbeiten. Genannt seien das vor 1961 fehlende Arzneimittelrecht, die ignorante Haltung der Politik gegenüber dem Thema Arzneimittelsicherheit, das Konsumverhalten der westdeutschen Gesellschaft oder das Hierarchiegefälle zwischen Ärzt_in und Patient_in. In diesem Sinne bietet der Band einen guten Überblick über das Thema „Contergan“.

Ganz wesentlich wurde der Blick auf den Vorfall bislang durch die Arbeiten von Beate Kirk für die Wissenschafts-, Medizin- und Pharmaziegeschichte (Der Contergan-Fall. Eine unvermeidbare Arzneimittelkatastrophe? Zur Geschichte des Arzneistoffes Thalidomid, 1999) und Willibald Steinmetz für die Politik- und Gesellschaftsgeschichte (Ungewollte Politisierung durch die Medien? Die ConterganAffäre, in: Weisbrod, Bernd (Hrsg.): Die Politik der Öffentlichkeit - Die Öffentlichkeit der Politik, 2003) geprägt. Dieses Bild ist durch den rezensierten Band, der sich ebenfalls auf diese Arbeiten bezieht, letztlich nicht grundlegend verändert worden. Die Stärke der vorliegenden Publikation ist die Ergänzung und der Ausbau schon bekannter grundsätzlicher Sachverhalte durch akribische Detailforschung sowie die anschließende Zusammenfassung der wissenschafts- und medizinhistorischen Sachverhalte. Mit dieser Grenzziehung sind auch schon die Schwächen des Bandes angesprochen. Sie lassen sich in zwei Punkten zusammenfassen.

Erstens wird Kulturgeschichte zwar im Kontext der Vorbedingungen des Skandals behandelt, von einer umfassenden Berücksichtigung kann allerdings nicht die Rede sein. So hätte man sich als Leserin gewünscht, etwas über die Opfer des Skandals zu erfahren und man fragt sich, warum hier kein eigener Beitrag unter Einbezug der Betroffenen aufgenommen wurde. Dieses Manko ist meines Erachtens vor dem Hintergrund von Debatten über das Empowerment von Patient_innen und Diversität besonders problematisch. Ferner fehlen zudem andere wichtige Beiträge, so beispielsweise über die Medizintechnik (Entwicklung von Prothesen) oder über die Verarbeitung des Skandals in den Medien.

Zweitens - und daran anschließend - verzichten die Autoren beziehungsweise nicht zuletzt auch die Herausgeber auf eine sorgfältige Analyse des Vorfalls. Dass es sich um mehr als einen von mehreren Arzneimittelskandalen handelte, die die Medizingeschichte kennt, wird in der Einleitung zwar erwähnt, aber diesem Umstand nicht gebührend Rechnung getragen. Das ist umso bedauerlicher, da der Band - zumindest teilweise - auch das Material liefert, um das Thema mit seinen breiteren Implikationen zu interpretieren. Ein Menetekel war „Contergan“ für zahlreiche Themen, die dann erst in den späten 1960er Jahren aufbrachen und die zum Teil bis heute aktuell geblieben sind: die Limitierungen der naturwissenschaftlichen Medizin, die Arrosion der ärztlichen Autorität, die Vernachlässigung von Patient_innen-Interessen, die Dominanz der pharmazeutischen Industrie, der Umgang mit ,Behinderung ‘ und generell mit Minderheiten in der Medizin. „Contergan“ als aufgeladener Begriff umgibt in diesen Punkten eine Aura - und an dieser Stelle sei der Vergleich gestattet - wie der Untergang der Titanic für Technikfolgen und Technikfolgeabschätzung. 
Das wird zwar angedeutet, aber man hätte in diesem Sinne mehr aus dem Thema machen können.

Insgesamt handelt es sich um eine gute Zusammenfassung des bisher Erreichten mit wichtigen und informativen zusätzlichen Forschungen. Dahingehend ist die Anschaffung für alle medizin- und wissenschaftshistorisch Interessierten und entsprechende Institutionen empfohlen. Allerdings beschleicht einen beim Lesen doch der Eindruck, dass die Chance verpasst wurde, eine umfassende Darstellung von „Contergan“ vorzulegen, die dem Anspruch des Bandes gerecht geworden wäre.

Funding Open Access funding enabled and organized by Projekt DEAL.

Open Access Dieser Artikel wird unter der Creative Commons Namensnennung 4.0 International Lizenz veröffentlicht, welche die Nutzung, Vervielfältigung, Bearbeitung, Verbreitung und Wiedergabe in jeglichem Medium und Format erlaubt, sofern Sie den/die ursprünglichen Autor(en) und die Quelle ordnungsgemäß nennen, einen Link zur Creative Commons Lizenz beifügen und angeben, ob Änderungen vorgenommen wurden.

Die in diesem Artikel enthaltenen Bilder und sonstiges Drittmaterial unterliegen ebenfalls der genannten Creative Commons Lizenz, sofern sich aus der Abbildungslegende nichts anderes ergibt. Sofern das betreffende Material nicht unter der genannten Creative Commons Lizenz steht und die betreffende Handlung nicht nach gesetzlichen Vorschriften erlaubt ist, ist für die oben aufgeführten Weiterverwendungen des Materials die Einwilligung des jeweiligen Rechteinhabers einzuholen.

Weitere Details zur Lizenz entnehmen Sie bitte der Lizenzinformation auf http://creativecommons.org/ licenses/by/4.0/deed.de. 